\title{
RANK AND ABELIAN RANK OF SYSTEMS OF EQUATIONS OVER THE FREE GROUP
}

\author{
by JOHN LAWRENCE
}

(Received 9th January 1996)

\begin{abstract}
Given a finite irredundant system of equations to be solved over the free group, one has four non-negative integers: the number of equations, the number of indeterminants, the rank of the system and the Abelian rank of the system. We show which four-tuples can actually occur.
\end{abstract}

1991 Mathematics subject classification: primary $20 \mathrm{E} 05$.

\section{Introduction}

Associated with a system of free group equations we have a quadruple of integers: the number of equations, the number of unknowns or variables, the rank of the system and the Abelian rank of the system. It is natural to ask exactly which quadruples are possible. Of course to make the number of equations meaningful, we must insist that the system be independent or irredundant. A complete description of the possible quadruples is given in this paper.

Let us first consider the analogous problem for Abelian group equations. Associated with an independent system of equations we have a triple of integers: the number of equations $(m)$, the number of variables $(n)$ and the Abelian rank $(a)$. The integers $m, n$ are nonnegative and a basic theorem states that $a=n-m$. This completely describes the possible triples.

As we shall see, the situation for nonabelian free groups is much more complicated.

\section{Basic concepts}

Let $E_{1}\left(X_{1}, \ldots, X_{n}\right), \ldots, E_{m}\left(X_{1}, \ldots, X_{n}\right)$ be words in the free group, $\mathbb{F}_{n}$, generated by $X_{1}, \ldots, X_{n}$. Let $\mathbb{F}_{\infty}$ be the countably generated free group. If $a_{1}, \ldots, a_{n} \in \mathbb{F}_{\infty}$, we say that $X_{1}=a_{1}, \ldots, X_{n}=a_{n}$ is a solution to the system of equations $E_{1}\left(X_{1}, \ldots, X_{n}\right)=1, \ldots, E_{m}\left(X_{1}, \ldots, X_{n}\right)=1$ if $E_{1}\left(a_{1}, \ldots, a_{n}\right)=\ldots=E_{m}\left(a_{1}, \ldots, a_{n}\right)=1$ in $\mathbb{F}_{\infty}$. This solution induces a group morphism $\varphi: \mathbb{F}_{n} \rightarrow \mathbb{F}_{\infty}$ which sends each $X_{i}$ to $a_{i}, i=1,2, \ldots, n$. The rank of the solution is the rank of the (free) subgroup of $\mathbb{F}_{\infty}$ 
generated by $\left\{a_{1}, \ldots, a_{n}\right\}$. The rank of the system of equations is the maximum rank of the solutions. If $\mathcal{E}$ is a system of equations we denote this rank by $r k \mathcal{E}$.

In the above discussion replace $\mathbb{F}_{\infty}$ by $\mathbb{A}_{\infty}$, the countably generated free Abelian group. In this case we have an Abelian rank of a solution and the Abelian rank of a system $\mathcal{E}$ of equations. If $\mathcal{E}$ is a system of equations, we denote this Abelian rank by $a b r k \mathcal{E}$.

Both the rank and Abelian rank of a system of equations depend only on the solution set in $\mathbb{F}_{\infty}$. Thus, two systems of equations that have the same solution set in $\mathbb{F}_{\infty}$ have the same rank and the same Abelian rank.

We say that a system of equations is irredundant (or independent) if the solution set (in $\mathbb{F}_{\infty}$ ) of the system is not equal to the solution set of any proper subset of the system. Subsystems of irredundant systems are irredundant.

In proving our result, we shall make frequent use of two important theorems.

Theorem (Schützenberger). The equation $[X, Y]=Z^{n} ; n>1$ has rank 1 .

Theorem (Lyndon-Schützenberger). The equation $X^{l} Y^{m} Z^{n}=1, l, m, n>1$, has rank 1 .

We define the commutator $[X, Y]=X^{-1} Y^{-1} X Y$ and the generalized commutator inductively by

$$
\left[X_{1}, \ldots, X_{n}\right]=\left[\left[X_{1}, \ldots, X_{n-1}\right], X_{n}\right] .
$$

See [3] for a discussion of these two results.

\section{The main result}

Suppose that we have a system $\mathcal{E}$ of $m$ equations in $n$ unknowns. Let $a=$ Abelian rank of $\mathcal{E}$ and $r=\operatorname{rank}$ of $\mathcal{E}$. Then $a, r, m$ and $n$ satisfy:

(0) $m$ and $n$ are positive integers; $a$ and $r$ are non-negative integers,

(1) $n \geq a \geq n-m$,

(2) $a \geq r$,

(3) $a \geq 1$ implies $r \geq 1$.

If, in addition, $\mathcal{E}$ is an irredundant system, then $a, r, m$ and $n$ also satisfy:

(4) $n>r$,

(5) $a=r$ implies $a=r=n-m$,

(6) $n \leq 2$ implies $a<n$,

(7) $m \geq n$ implies $a \neq 2$.

Note that (0) is straightforward. The condition that $m$ and $n$ both be positive is to avoid the trivial case. Condition (2) follows from the fact that the Abelianization of a 
free group of rank $n$ is a free Abelian group of rank, while (1) is a basic fact about equations over Abelian groups. Condition (3) holds because the free group of rank one is the same as the free Abelian group of rank one.

Condition (4) is basic to equations in groups [3, p. 95], while condition (6) follows from this along with the fact that rank one free groups are Abelian.

Condition (5) is due to the author [2, Theorem 5]. Condition (7) follows from (5) [2, Theorem 5].

Theorem. For any quadruple $(a, r, m, n)$ of integers satisfying conditions (0)-(7) there is an irredundant system of $m$ equations in $n$ variables with rank $r$ and Abelian rank $a$.

We prove the theorem in the next section.

\section{Proof of the main result}

Suppose that the integers $a, r, m$ and $n$ satisfy condition (0)-(7) of Section 3 . The proof is partitioned according to the value of these parameters.

$a=r-$ Consider the system $X_{1}=1, X_{2}=1, \ldots, X_{m}=1$. This has rank and Abelian rank equal to $n-m$. Note that $a=r=n-m$ by (5).

$$
\begin{array}{ll}
a>r, r=m=1 & - \text { This is Theorem 1. (Note that } n \geq a \geq n-1 \text { by (1).) } \\
a \geq r, r \geq 2 & - \text { This is Theorem 2. } \\
a>r, r=1, m>1 & \text { - This is Theorem 4. }
\end{array}
$$

Theorem 1. For each positive integer $n$ there exist two systems each consisting of a single equation in $n$ variables, each of the two equations with rank 1 , and one with Abelian rank $n$, the other with Abelian rank $n-1$.

Proof. Let the variables be $X_{1}, X_{2}, \ldots, X_{n}$. Let $C_{1}, C_{2}, \ldots, C_{n(n-1)}$ be an enumeration of the commutators $\left[X_{1}, X_{j}\right], 1 \leq i<j \leq n$.

Define two terms $T=T_{n(n-1)}$ and $T^{*}=T_{n(n-1)}^{*}$ inductively as follows.

First, $T_{1}=C_{1}$ and $T_{1}^{*}=X_{1}^{2} C_{1}$. Inductively, $T_{i+1}=T_{i}^{2} C_{i+1}$ and $T_{i+1}^{*}=T_{i}^{* 2} C_{i+1}$.

We claim that $T=1$ and $T^{*}=1$ satisfy the conditions given in the statement of the theorem.

First consider $T=1$. If $X_{1}=a_{1}, \ldots, X_{n}=a_{n}$ is a solution, we must show that the $a_{i}$ 's commute among themselves. From Schützenberger's Theorem, we see inductively that $\left[a_{i}, a_{j}\right]=1$ for all commutators, and this proves our claim. For $T^{*}=1$, we get in addition that $a_{1}=1$.

Theorem 2. Suppose that $m, n, a$ and $r$ are integers satisfying conditions (0)-(7). Suppose further that $r \geq 2$ and $a>r$. Then there exists an irredundant system of $m$ group equations in $n$ variables with rank $r$ and Abelian rank $a$.

Proof. Let the variables be $X_{1}, \ldots, X_{n}$. Note that $a \geq r+1 \geq 3$. For each integer $i$ satisfying $1 \leq i \leq m$, construct the generalized commutator 


$$
B_{i}=\left[X_{r+1}, X_{1} X_{2} \cdots X_{r}, X_{1}^{2} X_{2} \cdots X_{r}, \cdots, X_{1}^{i-1} X_{2} \cdots X_{r}, X_{1}^{i+1} X_{2} \cdots X_{r}, X_{1}^{m} X_{2} \cdots X_{r}\right]
$$

Let

$$
A_{i}= \begin{cases}X_{a+i}^{2} & \text { if } 1 \leq i \leq n-a \\ 1 & \text { if } n-a \leq i \leq m\end{cases}
$$

Finally, let $C_{1}, C_{2}, \ldots, C_{l}$ be a listing of the commutators of the form $\left[X_{i}, X_{j}\right]$ with $1 \leq i \leq a$ and $r+2 \leq j \leq a$.

We construct the group term $E_{k}$ for the $k^{\prime}$ th equation in our system inductively as follows. Let $T_{1}=\left(\left(A_{K}\right)^{2} B_{k}\right)^{2} C_{1}$ and let $T_{j+1}=T_{j}^{2} C_{j+1}$. Then $E_{k}=T_{l}$. We claim that the system $\mathcal{E}=\left\{E_{1}=1, E_{2}=1, \ldots, E_{m}=1\right\}$ satisfies our conditions.

Certainly there are $m$ equations in $n$ unknowns. When Abelianized these $m$ equations become $X_{a+1}^{2}=1, \ldots, X_{n}^{2}=1,1=1, \ldots, 1=1$, so the Abelian rank of $\mathcal{E}$ is $a$. Moreover, the system is irredundant as $X_{r}=\left(X_{1}^{j} X_{2} \cdots X_{r-1}\right)^{-1}$ is a solution to the $i$ 'th equation for $i \neq j$, but is not a solution to the $j$ 'th equation. It remains only to check the rank of the system.

As $X_{r+1}=X_{r+2}=\cdots=X_{n}=1$ is a solution to the system, the rank of $\mathcal{E}$ is at least $r$. By Schützenberger's Theorem, $C_{i}=1, i=1,2, \ldots, l$; hence $X_{j}$ commutes with $X_{i}$ for all $1 \leq i \leq a$ and $r+2 \leq j \leq a$. Moreover, $B_{k}=1$ and $A_{k}=1$. The latter fact tells us that $X_{i}=1$ for $a+1 \leq i \leq n$. Therefore $X_{j}(r+2 \leq j \leq a)$ commutes with all $X_{i}$ in any solution. Thus the rank of $\mathcal{E}$ is at most the rank of $B_{k}=1$, which is $r$.

This completes the proof of the theorem.

Theorem 3. Let $A(X, Y, Z)$ and $B(X, Y, Z)$ be group terms in three variables $X, Y, Z$ such that for some nonzero integer $m,\left[A\left(X, X^{-m}, Z\right), B\left(X, X^{-m}, Z\right)\right]$ is not identically 1 in the free group. Then for suitably large $n$, the equation

$$
[A(X, Y, Z), B(X, Y, Z)]=X^{m 2^{n}} Y^{2^{n}}
$$

has rank 1.

Proof. To prove this theorem we use theorems on unification of group equations. The equation certainly has Abelian rank 2. If the rank of the equation is also 2 , then it is unitary in the variety of groups and thus unitary in the nilpotent class $k$ groups [2, Theorem 1].

Let $k$ be the smallest integer such

$$
\left[A\left(X, X^{-m}, Z\right), B\left(X, X^{-m}, Z\right)\right]
$$

is not identically 1 in nilpotent class $k$ groups. Then $k>1$. In nilpotent class $k-1$ groups, we have the equation

$$
[A(X, Y, Z), B(X, Y, Z)]=X^{m 2^{n}} Y^{2^{n}}
$$


which has solution $Y=X^{-m}$. This is the general solution in nilpotent class $k-1$ groups [1, Proposition 3]. The general solution (in the unitary case) in nilpotent class $k$ groups is given by

$$
X=U P_{1}, \quad Y=U^{-m} P_{2},
$$

where $P_{2}$ and $P_{2}$ are products of basic commutators of weight $k$ satisfying

$$
\left[A\left(U P_{1}, U^{-m} P_{2}, Z\right), B\left(U P_{1}, U^{-m} P_{2}, Z\right)\right]=P_{1}^{m 2^{n}} P_{2}^{2^{m}}
$$

By hypothesis, the left side is a non-empty product of powers of basic commutators in $U$ and $Z$. By the main theorem (Theorem 5) in [1], the equation is unitary only if $2^{n}$ divides one of the nonzero exponents on the left side. But the exponents on the left side do not depend on $n$, so for suitable large $n$ this is not possible.

Remarks. The above result does not hold if we drop the condition "suitably large $n$ ".

Let $A(X, Y, Z)=X^{-m 2^{n}}$ and $B(X, Y, Z)=Z$. Then the equation $\left[X^{-m 2^{n}}, Z\right]=X^{m 2^{n}} Y^{2^{n}}$ has solution $Y=Z^{-1} X^{-m} Z$, which has rank 2 .

Implicit in the proof is the fact that the extraction of l'th roots is unique in free nilpotent groups.

Theorem 4. Suppose that $m$ and a are integers both greater than 1. Suppose further that $n$ is a positive integer such that $m, n, a$ and $r=1$ satisfy the conditions (0)-(7). Then there is an irredundant system of m equations in $n$ unknowns with rank 1 and Abelian rank a.

Proof. If $n \leq 2$, then $a<n$, so, as $a \geq 2$, we have $n \geq 3$.

We first define a sequence of integers $1=n_{1}<n_{2}<\cdots<n_{m}$ and a sequence of terms $W_{1}=W_{1}(X, Y), W_{2}=W_{2}(X, Y), \ldots, W_{m}=W_{m}(X, Y)$, in variables $X$ and $Y$, inductively as follows.

The word $W_{1}(X, Y)=X^{4} Y^{2}$. Choose $n_{i+1}\left(>n_{i}\right)$ large enough such that

$$
\left[X^{2} Z^{j+1}, W_{1}, \ldots, W_{j-1}, W_{j+1}, \ldots, W_{i}\right]=X^{(i+1) 2^{n_{i+1}}} Y^{2^{n_{1+1}}}
$$

has rank 1 (for each $j<i$ ) and $\left[X^{2} Z^{j+1}, W_{1}, \ldots, W_{i}\right]=X^{(i+1) 2^{n_{i+1}}} Y^{2^{n_{i+1}}}$ has rank 1 (for all $m \geq j \geq i)$. It is here that we use Theorem 3 .

Let $W_{i+1}=X^{(i+1) 2^{n_{i+1}}} Y^{2^{n_{1+1}}}$. For each $j, 1 \leq j \leq m$, let $E_{j}=\left[X^{2} Z^{j+1}, W_{1}, \ldots, W_{j-1}, W_{j+1}, \ldots, W_{m}\right]$. We claim that the only rank 2 solution of $E_{j}=1$ occurs when $X^{2} Z^{j+1}=1$ or $W_{1}=1$ or $W_{2}=1$ or $\cdots$ or $W_{m}=1$.

First note that if, under some substitution in the free group, $X$ and $Y$ commute, then they are powers of some common element $U$. Substituting in $E_{j}$ we get

$$
\left[U^{S_{0}} Z^{j+1}, U^{s_{1}}, U^{S_{2}}, \ldots, U^{S_{m}}\right]=1
$$

This gives a rank 2 solution only if $U^{S_{0}} Z^{j+1}=1$ or $U^{S_{1}}=1$ or $\cdots$ or $U^{S_{m}}=1$. 
Now assume that, under a substitution, $X$ and $Y$ do not commute. As $\left[X^{2} Z^{j+1}, W_{1}, \ldots, W_{m-1}\right]$ and $W_{m}$ commute,

$$
\left[X^{2} Z^{j+1}, \ldots, W_{m-1}\right]=U^{S}
$$

and

$$
X^{(m+1) 2^{n+1}} Y^{2^{n+1}}=W_{m}=U^{t}
$$

for some element $U$ and for some integers $s$ and $t$. By the Lyndon-Schützenberger Theorem, $|t|=1$ and by the Schützenberger Theorem, $U=1$ or $|s|=1$. If $U=1$, then we use this as an inductive reduction. If $|s|=1$, then $s= \pm t$, so

$$
\left[X^{2} Z^{j+1}, \ldots, W_{m-1}\right]=W_{m}^{ \pm 1} .
$$

By our construction, this equation has rank 1.

This completes the proof of our claim.

To prove our theorem, consider first the case when $a \geq 3$. We thus have $a+1>3$ and $m \geq n-a$.

Define $A_{i}=A_{i}\left(X_{4}, \ldots, X_{n}\right), i=1,2, \ldots, n$, by

$$
A_{i}= \begin{cases}X_{n-i+1} & \text { if } n \geq i \geq a+1 \\ 1 & \text { otherwise }\end{cases}
$$

Let $C_{1}, C_{2}, \ldots, C_{t}$ be a list of the commutators $\left[X_{i}, X_{j}\right], 1 \leq i<j \leq n$, where $j>3$. Let $B=\left(\left(\left(X_{n}^{2} C_{1}\right)^{2} C_{2}\right)^{2} \cdots\right)^{2} C_{t}$. Our equations are

$$
\begin{gathered}
B^{2} E_{1}\left(X_{1}, X_{2}, X_{3}\right)=1 \\
A_{2}^{2} E_{2}\left(X_{1}, X_{2}, X_{3}\right)=1 \\
\cdots \\
A_{m}^{2} E_{m}\left(X_{1}, X_{2}, X_{3}\right)=1
\end{gathered}
$$

where $E_{i}$ is the term in three variables constructed earlier.

We have $m$ equations in $n$ variables. As $X_{2}^{-j}=X_{3}, X_{4}=X_{5} \cdots=X_{n}=1$ is a solution of all but the $j^{\prime}$ th equation, the system is irredundant.

Clearly the Abelian rank of the system is $a$, so it remains to prove that the system has rank 1 .

If under any substitution $X_{i} \neq 1$ for $i>3$, then all the entries commute among themselves and this gives us rank 1 . Therefore assume that $X_{i}=1$ for $i>3$. We are left with the system

$$
\begin{gathered}
E_{1}\left(X_{1}, X_{2}, X_{3}\right)=1 \\
\ldots \\
E_{m}\left(X_{1}, X_{2}, X_{3}\right)=1 .
\end{gathered}
$$


By our claim this has rank 2 only if $X_{1}^{-2}=X_{3}^{i+1}$ and $X_{2}^{-1}=X_{1}^{j}$ for some $i$ and $j$ between 1 and $n$. It follows that $X_{1}, X_{2}$ and $X_{3}$ commute among themselves, thus the rank is at most 1 .

To complete the proof of the theorem we now consider the case where $a=2$. It follows that $n=m+1$ or $n=m+2$. If $n=m+1$, consider the system of equations

$$
\begin{aligned}
{\left[X_{1}, X_{2}\right] } & =1 \\
X_{3} & =1 \\
\cdots & \\
X_{n} & =1 .
\end{aligned}
$$

If $n=m+2$, consider the equations

$$
\begin{aligned}
X_{1}^{2}\left[X_{2}, X_{3}\right] & =1 \\
X_{4} & =1 \\
\cdots & \\
X_{n} & =1 .
\end{aligned}
$$

These systems satisfy our conditions. This completes the proof of the theorem.

\section{REFERENCES}

1. M. Albert and J. Lawrence, Unification in Varieties of groups: Nilpotent groups, Canad. J. Math. 46 (1994), 1135-1149.

2. J. LAWRENCE, On the unification of group equations.

3. R. Lyndon, Equations in groups, Bol. Soc. Brasil. Mat. 11 (1980), 79-102.

Department of Pure Mathematics

UNIVERSITY OF WATERLOO

WATERLOO

ONTARIO

Canada N2L 3G1 